\title{
Control Strategies and Design Guidelines for Planar Latch-less Metamorphic Robots Based on Analysis of Dynamics
}

\author{
Ashish Deshpande \\ adeshpan@umich. edu \\ Univ. of Michigan \\ Ann Arbor, MI, USA
}

\author{
Siddhartha S. Srinivasa \\ siddhartha.srinivasa@intel.com \\ Intel Research \\ Pittsburgh, PA, USA
}

\author{
Padmanabhan Pillai \\ padmanabhan.s.pillaidintel.com \\ Intel Research \\ Pittsburgh, PA, USA
}

\begin{abstract}
Modular robotic systems with no fixed mechanical contacts have the ability to adopt and reconfigure very rapidly, but are very difficult to control dynamically. Moving module solely with electro-magnetic or -static forces can lead to unwanted slipping or even loss of contact. This paper presents a strategy to design controller for such modules based on the limits derived by combining the contact constraints and the actuator saturation. We demonstrate the design of a simple but effective controller for two module motions. We also present guidelines for the design of the modules based on the controller limitations.
\end{abstract}

\section{INTRODUCTION}

A metamorphic system is comprised of an ensemble of modular units that are connected to one another. Modules are usually capable of motion relative to each other, thereby moving the entire system. Metamorphic systems are especially attractive due to this modular construction as they can achieve a rich set of complex motions by the collective actuation of the individual simple modules. Since reconfiguration of the entire system is achieved by the local motion of numerous individual modules about each other, the design of robust mechanisms and algorithms for local motion are crucial for the function and efficiency of the system.

Physical interactions between modules are threefold: when not moving, individual modules need to stay connected to each other; modules must exert forces on each other to cause motion; and modules in motion must eventually stop and latch onto other modules. Traditionally, these interactions have been realized by means of latches which comprise of mechanical structures that are actuated to engage and disengage modules, such as the MTran [11], ATran [6], CORNO [8] and Polybot [18] systems. Latching mechanisms have the advantage of a guaranteed strong mechanical lock once engaged. However, they suffer from a few problems. First, latches comprise of, sometimes intricate, moving parts that need to be manufactured and assembled for each module. Second, repeated actuation can lead to wear and tear and eventual failure of the latches. Third, latches merely connect and disconnect thus needing additional mechanism to actuate module movements.

Claytronics [4] envisions million-module self reconfigurable robot ensembles capable of reproducing moving, physical 3-D shapes. To facilitate module manufacturing and speedy reconfiguration, this system uses modules which bond to each other without engaging any mechanical latches or hooks [9]. At a macro-scale Claytronic modules, called catoms, employ a number of electromagnets arranged around the spherical or cylindrical module as depicted in Figure 1(a). At the micro-scale we envision actuation using a similar arrangement of electrostatic actuators. Latch-less systems offer numerous advantages. First, the latching mechanism has no moving parts as engagement and disengagement are achieved by just changing the polarity of the electromagnets. Second, 'Force-at-a-distance' mechanisms are used not only to adhere but also to actuate the modules by using adjacent electromagnets. Third, each electromagnet provides a continuous area of contact points between modules.

The price paid for all of the above advantages is in algorithmic complexity. The lack of fixed contact between the modules poses the challenging problem of modules much more likely to slide past each other, or worse, lose contact and disengage as they move. Successful motion, thus, requires the careful orchestration of electromagnetic forces at multiple contacts, in addition to motion planning challenges. Another issue with latch-less, cylindrical, magnetic modular robots is that we do not have the luxury of velocity-controlled mechanical joints, independent module actuation, or selflimiting step-wise actuation mechanisms, and must consider dynamics in all actuation.

In this paper we present a methodology to design control strategies to achieve desired module motions without slip or loss of contact. There are three important goals of this paper. First, we demonstrate a strategy to combine the contact constraints, namely, no-slip or no loss of contact condition and the actuator limits, namely, the strength of magnets to determine the limits for controller design. Secondly, we demonstrate design of a simple but effective controller using these limits. And finally, we demonstrate how different design choices affect these controller limits and thus lead to useful design guidelines. Tools exist in the mobile robotics [3] and the manipulation [17] literature for control synthesis under dynamic contact constraints such as slip and loss of contact. In this paper we present the adaptation of these tools to metamorphic systems. Specific challenges such as modeling the magnetic forces, modeling the ground friction forces are addressed here.

We focus our discussion on the reconfiguration of an ensemble of cylindrical modules in a planar lattice. Here, a module moves from one lattice position to an empty position by rolling against a neighbor. This is an important class of movement is used as the base motion primitive 
for a variety of scalable, distributed shape reconfiguration and planning algorithms, including hole motion [13] and templated hierarchical planning [2] as shown in Figure 3. To make the analysis of a catom rolling against a large ensemble tractable, we use a two catom model, fixing one as immovable, closely approximating the neighbor attached to a large mass of other catoms (Figure 1). We separately analyze the stopping condition when a moving catom comes to rest against neighbors in its new lattice position, ensuring the impact does not cause slipping or loss of contact at the moving interface. These analyses fully define the dynamics of lattice-style reconfiguration of planar, cylindrical catoms employing force-at-a-distance actuation and no mechanical latching mechanisms.

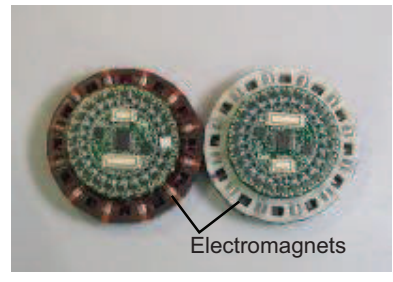

(a) An overhear view of two Catoms

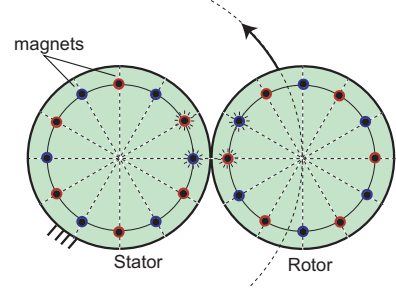

(b) Sketch of two interacting Catoms

Fig. 1. Two interacting Catoms: a stator Catom fixed to ground and a rotor Catom revolving around stator.

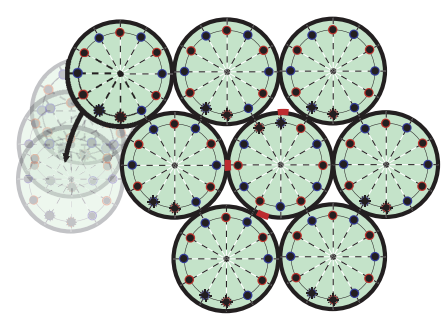

Fig. 2. Catom flies off: as we try to control the rotor motion around the stator, the rotor flies off at high velocity.

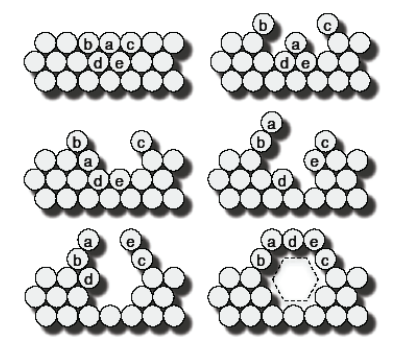

(a) Sketch of hole motion with catoms [13]

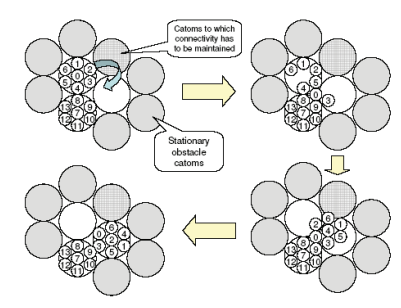

(b) Sketch of kinematic plans with catoms [2]
In Section II we present the detailed model of two interacting catoms including the magnetic force model. We also present a mathematical condition to check for the slipping between two catoms. In the same section we also present a simple control strategy for two interacting catoms which does not give good results. In Section III we present a methodology to combine the friction cone limits with magnetic force limits and we also present control strategies based on the combined limits. In Section III-D we present a simple but effective controller for two catom motion based on the strategies presented in III. In Section IV we present some general design guideline based on the controller limits. Finally, in Section V we conclude and present ideas for future work.

\section{Two InTERACTING CATOMS}

Figure 1(a) shows the hardware of two interacting catoms and Figure 1(b) shows the corresponding cartoon. The catom motions are achieved by modulating the strength of the magnets around the periphery of the catoms. For the purpose of our analysis, we assume that the magnets are at a depth of $d_{m}$ from the catom surface.

The analysis of the motion of one catom in the ensemble can be simplified by assuming that the ensemble is sufficiently large so that it does not move in response to the motion of the single catom. In that case, the problem can be simplified to that of two interacting catoms with one of the catoms fixed to ground (representing the ensemble) and other other catom free to move in the plane. We want to design a control strategy to get the moving catoms (rotor) revolve around the fixed catom (stator) by a desired angle.

Since there is no latching mechanism that secures the two catoms to each other, there is a possibility of slip between the catoms, which is undesirable as it leads to misalignment between the magnets, or worse, there is a possibility of loss of contact between the catoms. We want to design a controller to rotate the rotor around the stator without slip or loss of contact.

\section{A. Dynamics of Two catoms}

We derive they dynamic equations of planar motion of two interacting catoms. We assume a Coulomb friction model at the contact between the two catoms and also at the surface contact between the ground and the rotor. We also assume that the catoms are aligned at the start of motion. Our goal is to keep them aligned throughout the motion.

The physical parameters for the catoms are based on the hardware system and they are: mass, $m=0.2 \mathrm{Kg}$, catom radius, $R=2 \mathrm{~cm}$, magnet radius, $R_{m}=0.8 R$, gravity, $g=$ $9.8 \mathrm{~m} / \mathrm{s}^{2}$, number of magnet, $n_{m}=18$, coefficient of friction between the rolling catoms, $\mu_{c}=0.5$, coefficient of friction between the ground and the catom $\mu_{g}=0.5$, angular distance between the magnets, $\phi=\frac{2 \pi}{n_{m}}$

The condition that the catoms must not slip or lose contact can be expressed in terms of a friction cone constraint. This idea is used previously by Srinivasa et al. [16] for robotic

Fig. 3. Examples of catom motion plans. 


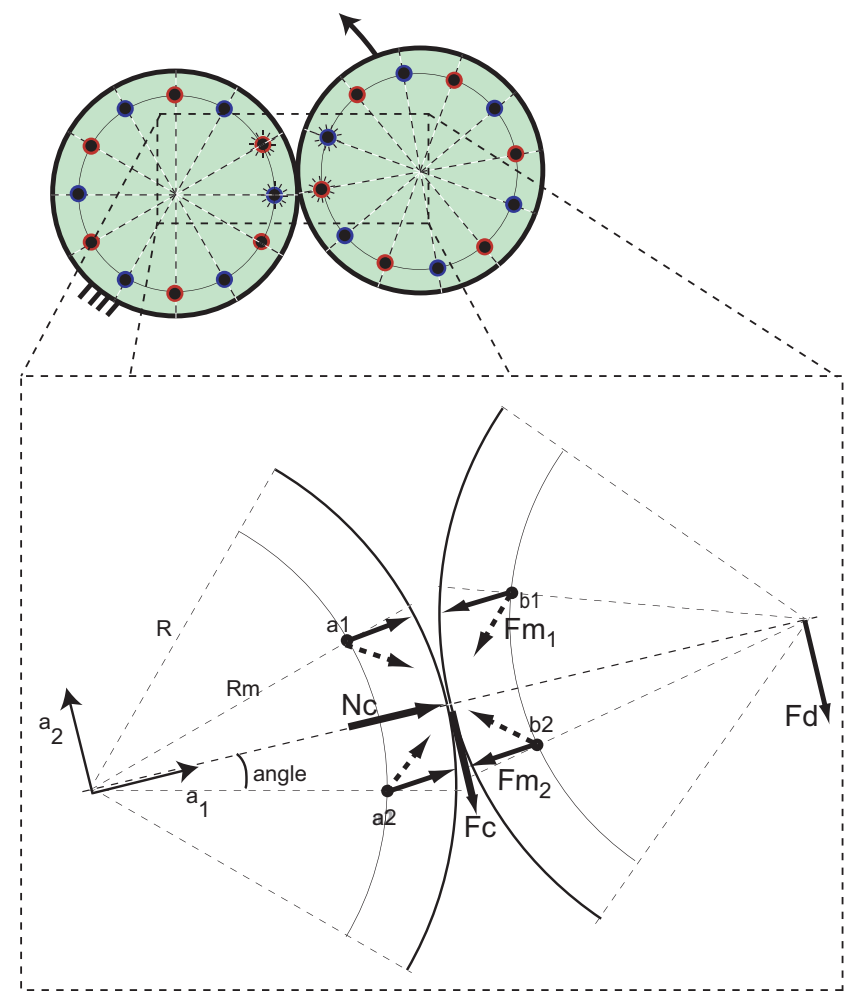

Fig. 4. Force vectors in the system two interacting catoms. Zoomed-in view shows two active pair of magnets: the normal force and the tangential force are at the point of contact $\mathrm{C}$ and angle is $\theta$.

manipulation problem and also by Deshpande and Luntz [3] for mobile robot applications. The basic principle is that, to avoid slipping, the resultant contact force, at the point of contact $\mathrm{C}$ between the catoms, must lie inside a cone defined by angle $\alpha$ where $\alpha=\arctan \left(\mu_{c}\right) ; \mu_{c}$ is the coefficient of friction between the rolling catoms.

The system of two interacting catoms with one catom fixed to ground has one degree of freedom (DOF) and we can define it as the angle $\theta$ that the line joining the two centers makes with the horizontal. To check for the no-slip condition we need to determine the contact forces between the two catoms and these forces depend on the applied magnetic forces as well as the dynamics of catoms. One way to bring these forces into the analysis is to introduce extra degrees of freedom in analysis and then constrain these extra degrees of freedom. This is similar to the idea of Lagrange Multiplier [5] or the idea of pseudo robots introduced by [3]. Since we want to determine the normal and the tangential forces at the point of contact, to check for friction cone constraint, we introduce two extra degrees of freedom: $x$ and $y$ : which are the $\mathrm{x}$-location and the $\mathrm{y}$-location of the center of mass of the rotor, respectively. Thus the set of generalized coordinates is: $\mathbf{q}=[x, y, \theta]^{T}$. We derive the equations of motions of this system using the Kane's dynamics methods[7] and they are:

$$
M \ddot{\mathbf{q}}=G_{c} \mathbf{f}_{\mathbf{c}}+G_{e 1} \mathbf{F}_{\mathbf{d}}+G_{e 2} \mathbf{F}_{\mathbf{m}}
$$

subject to the rolling contact constraint:

$$
\begin{aligned}
G^{T} \dot{\mathbf{q}} & =0 \\
G^{T} & =\left[\begin{array}{ccc}
1 & 0 & 2 R \sin (\theta) \\
0 & 1 & -2 R \cos (\theta)
\end{array}\right]
\end{aligned}
$$

and $\mathbf{f}_{\mathbf{c}}$ is the contact force matrix $\mathbf{f}_{\mathbf{c}}=\left[N_{c}, F_{c}\right]^{T}$. We have defined a rotating frame A with unit vectors $\hat{\mathbf{a}}_{1}, \hat{\mathbf{a}}_{2}, \hat{\mathbf{a}}_{3}$ such that $\hat{\mathbf{a}}_{\mathbf{1}}$ is aligned with the line joining the two centers. Thus, $\hat{\mathbf{a}}_{1}=\cos (\theta) \hat{i}+\sin (\theta) \hat{j}$ and $\hat{\mathbf{a}}_{2}=-\sin (\theta) \hat{i}+\cos (\theta) \hat{j}$. The contact force vectors are: $\mathbf{N}_{\mathbf{c}}=N_{c} \hat{\mathbf{a}}_{1}$ and $\mathbf{F}_{\mathbf{c}}=F_{c} \hat{\mathbf{a}}_{2} \cdot \mathbf{F}_{\mathbf{d}}$ is the reaction force as the rotor slides along the ground. We model it as a force applied at the center of the rotor, with magnitude $F_{d}=\mu_{g} \operatorname{Sign}(\dot{\theta}) \mathrm{mg}$, in the direction opposite to the direction of motion: $\mathbf{F}_{\mathbf{d}}=F_{d} \hat{\mathbf{a}}_{\mathbf{1}} . M$ is the mass matrix as defined below:

$$
M=\left[\begin{array}{ccc}
m & 0 & 0 \\
0 & m & 0 \\
0 & 0 & \frac{m R^{2}}{2}
\end{array}\right],
$$

$G_{c}$ is the grasp map of the contact forces

$$
G c=\left[\begin{array}{cc}
\cos (\theta) & -\sin (\theta) \\
\sin (\theta) & \cos (\theta) \\
0 & R
\end{array}\right],
$$

and, $G e_{1}$ and $G e_{2}$ are as follows:

$$
\begin{gathered}
G e_{1}=\left[\begin{array}{c}
\sin (\theta) \\
-\cos (\theta) \\
0
\end{array}\right], \\
G e_{2}=\left[\begin{array}{cc}
\cos (\theta) & \cos (\theta) \\
\sin (\theta) & \sin (\theta) \\
R_{m} \sin (\phi-\theta) & R_{m} \sin (\theta)
\end{array}\right] .
\end{gathered}
$$

1) Magnetic Force Model: Our magnetic force model is based on the assumption of two point charges generating the magnetic field. We model the magnitude of the magnetic force between two magnets of the two catoms as inversely proportional to the cube of the distance between the magnets and the direction of the force along the line joining the two magnets. If $\mathbf{r}$ is the vector connecting two magnetic points, then

$$
\mathbf{F}_{\mathbf{m} i}=F m_{i} \hat{\mathbf{r}}_{i}=\frac{K_{\max } K c}{\left\|\mathbf{r}_{i}\right\|^{3}} \hat{\mathbf{r}}_{i}
$$

, where $K_{\max }$ is a magnet constant and for our hardware system is equal to $10^{-5} \mathrm{~N}-\mathrm{m}^{3}$ and $K_{c}$ is the control parameter for controlling the magnet force. Figure 4 shows the magnet forces at point $a_{1}, a_{2}, b_{1}$ and $b_{2}$. The dotted vectors represent the forces between the cross magnet pairs. The vectors of these forces $\mathbf{F}_{\mathbf{m}}$ are derived as follows:

$$
\begin{aligned}
& \mathbf{r}_{1 \mathbf{1}}=\mathrm{d}\left(a_{1} b_{1}\right) \hat{\mathbf{a}}_{1}=\left[2 R-2 R_{m} \cos (\phi-\theta)\right] \hat{\mathbf{a}}_{1}, \\
& \mathbf{r}_{\mathbf{1 2}}=\mathrm{d}\left(a_{1} b_{2}\right) \mathbf{a}_{\mathbf{1}} \hat{\mathbf{b}}_{\mathbf{2}}=\left[2 R-2 R_{m} \cos (\phi-\theta)\right] \mathbf{a}_{\mathbf{1}} \hat{\mathbf{b}}_{\mathbf{2}}, \\
& \mathbf{r}_{\mathbf{1 1}}=\mathrm{d}\left(a_{1} b_{1}\right) \mathbf{a}_{\mathbf{2}} \hat{\mathbf{b}}_{\mathbf{1}}=\left[2 R-2 R_{m} \cos (\phi-\theta)\right] \mathbf{a}_{\mathbf{2}} \hat{\mathbf{b}}_{\mathbf{1}}, \\
& \mathbf{r}_{\mathbf{2 2}}=\mathrm{d}\left(a_{2} b_{2}\right) \hat{\mathbf{a}}_{1}=\left[2 R-2 R_{m} \cos (\theta)\right] \hat{\mathbf{a}}_{1},
\end{aligned}
$$




$$
\mathbf{F}_{\mathbf{m}}=\left[\begin{array}{l}
F_{m 1} \\
F_{m 2}
\end{array}\right]=D_{m a t} K_{m a t},
$$

where,

$$
\begin{aligned}
D_{\text {mat }} & =\left[\begin{array}{ll}
\frac{K \max }{\left\|\mathrm{d}\left(a_{1} b_{1}\right)\right\|^{3}} & \frac{K \max }{\| \frac{\mathrm{d}\left(a_{1} b_{2}\right) \|^{3}}{}} \\
\frac{K m a x}{\left\|\mathrm{~d}\left(a_{2} b_{1}\right)\right\|^{3}} & \frac{K m a x}{\left\|\mathrm{~d}\left(a_{2} b_{2}\right)\right\|^{3}}
\end{array}\right], \\
K_{\text {mat }} & =\left[\begin{array}{l}
K c_{1} \\
K c_{2}
\end{array}\right] .
\end{aligned}
$$

\section{Control Synthesis For CATOM Motion}

Based on the model of motion dynamics for two catoms we want to design controllers to achieve desired motion of the rotor from the initial state to the final state. While following the motions we want to make sure that catoms do not lose contact and do not slip. These conditions can be represented in terms of kinematic constraints (given by Equation 2) and constraints on the contact forces at point C. Introduction and constraining of degrees of freedom as shown in the earlier section does allow for determination the contact forces $\mathrm{f}_{\mathrm{c}}$ under the dynamic conditions.

One strategy for designing controller is to design feedback control law for the desired angle of revolution of the mover and then employ a check in terms of kinematics and contact cone constraints for each time step. We employed such strategy with a simple PID controller but it failed to a keep the contact forces inside the friction cone limit. We believe that the reason for the failure of such a control strategy is that we only react when cone constraints are close to be violated and there is no consideration for the speed at which cone constraint limits are approached.

To this end we propose a novel strategy. Our idea is to combine the kinematics constraints and contact force constraints, determine a set of allowable motions based on the combined constraints and then design a control law that executes desired motions from the allowable motions. This idea is adapted from the analysis presented by Srinivasa et al. for the robotic manipulation problems [16], [17] and by Deshpande and Luntz for the mobile robot applications [3].

In this section we start out by showing a strategy to combine kinematic and contact force constraints. We combine the constraints by first transforming all the constraints in the catom acceleration space. This results in a allowable motion region represented in the catom 'angle - angular velocity angular acceleration' space. Lets consider the equation of motion of the system:

$$
M \ddot{\mathbf{q}}=G_{c} \mathbf{f}_{\mathbf{c}}+G_{e 1} \mathbf{F}_{\mathbf{d}}+G_{e 2} \mathbf{F}_{\mathbf{m}} .
$$

We can re-write it as:

$$
\ddot{\mathbf{q}}=M^{-1} G_{c} \mathbf{f}_{\mathbf{c}}+M^{-1} G_{e 1} \mathbf{F}_{\mathbf{d}}+M^{-1} G_{e 2} \mathbf{F}_{\mathbf{m}} .
$$

The kinematic constraints for this system are: $G^{T} \dot{\mathbf{q}}=0$. Taking the time derivative of the kinematic constraints give:

$$
\dot{G}^{T} \dot{\mathbf{q}}+G^{T} \ddot{\mathbf{q}}=0
$$

Substituting $\ddot{\mathbf{q}}$ from 11 gives:

$\dot{G}^{T} \dot{\mathbf{q}}+G^{T}\left(M^{-1} G_{c} \mathbf{f}_{\mathbf{c}}+M^{-1} G_{e 1} \mathbf{F}_{\mathbf{d}}+M^{-1} G_{e 2} \mathbf{F}_{\mathbf{m}}\right)=0$

$$
\begin{array}{r}
\dot{G}^{T} \dot{\mathbf{q}}+G^{T} M^{-1} G_{e 1} \mathbf{F}_{\mathbf{d}} \\
+G^{T} M^{-1} G_{e 2} D_{m a t} K_{m a t}=-G^{T} M^{-1} G_{c} \mathbf{f}_{\mathbf{c}}
\end{array}
$$

Equation 12 gives the relationship between the contact forces $\mathbf{f}_{\mathbf{c}}$ and actuation forces defined by $\left[K c_{1}, K c_{2}\right]^{T}$ for a given $\{\mathbf{q}, \dot{\mathbf{q}}\}$. Lets define $\tilde{G}:=G^{T} M^{-1} G_{c}$ and we can re-write Equation 12 as ${ }^{1}$ :

$$
\begin{array}{r}
-\tilde{G}^{-1}\left(\dot{G}^{T} \dot{\mathbf{q}}+G^{T} M^{-1} G_{e 1} \mathbf{F}_{\mathbf{d}}+\right. \\
\left.G^{T} M^{-1} G_{e 2} D_{\text {mat }} K_{m a t}\right)=\mathbf{f}_{\mathbf{c}}
\end{array}
$$

So far we have combined the kinematic constraints on the catoms with the dynamics of the catoms and we have $\mathbf{f}_{\mathbf{c}}$ explicitly appearing in Equation 13. Now we can impose the 'no slip' and 'no loss of contact' conditions by constraining $\mathbf{f}_{\mathrm{c}}$ determined as above. As mentioned earlier we set up a friction cone constraint which says that is that $f_{c}$ must lie inside the friction cone defined by the coefficient of friction, and represented by $\mathcal{F}$, at the point of contact $C$ i.e. $\mathrm{f}_{\mathbf{c}} \in \mathcal{F}$. This constraint can be represented mathematically as follows.

\section{A. Representation of Friction Cone Constraint}

The cone condition can be specified mathematically as what is called the 'face normal representation'. The 2D version of this representation utilized two unit vectors $\mathbf{n}_{\mathbf{1}}$ and $\mathbf{n}_{2}$ each normal to one edge of the $2 \mathrm{D}$ friction cone. The friction cone satisfaction condition for a contact force vector (e.g. g) is then set up as follows:

$$
\mathcal{F}=\left\{\mathbf{g}: N \cdot \mathbf{g} \leq \mathbf{0}, N=\left[\begin{array}{ll}
\mathbf{n}_{\mathbf{1}} & \mathbf{n}_{\mathbf{2}}
\end{array}\right]^{T}\right\}
$$

Thus the friction cone condition $\mathbf{f}_{\mathrm{c}} \in \mathcal{F}$ can be written as N. $\mathbf{f}_{\mathrm{c}} \leq \mathbf{0}$, where

$$
N=\left[\begin{array}{cc}
-\sin (\alpha) & \cos (\alpha) \\
-\sin (\alpha) & -\cos (\alpha)
\end{array}\right]
$$

$\alpha=\arctan \left(\mu_{c}\right)$, and $\mu_{c}$ is coefficient of friction at the contact point $\mathrm{C}$.

\section{B. Limits on Kcs and $\ddot{\theta}$}

We can incorporate the face normal representation of the friction cone condition, N. $\mathbf{f}_{\mathbf{c}} \leq \mathbf{0}$, with Equation 13 as follows:

$$
\begin{array}{r}
-\mathbf{N} \tilde{G}^{-1}\left(\dot{G}^{T} \dot{\mathbf{q}}+G^{T} M^{-1} G_{e 1} \mathbf{F}_{\mathbf{d}}+\right. \\
\left.G^{T} M^{-1} G_{e 2} D_{m a t} K_{m a t}\right) \leq \mathbf{0}
\end{array}
$$

Above equation gives us the linear inequalities in terms of $K c_{1}$ and $K c_{2}$ corresponding to the friction cone condition. We can combine these with the min-max limits on $K c_{1}$ and $K c_{2}$ to determine the region of allowable values. Figure 5 shows intersection of the min-max limits of $K c \mathrm{~s}$ with inequalities represented by lines L1 and L2 for $\theta=0.2$ $\mathrm{rad}, \dot{\theta}=1 \mathrm{rad} / \mathrm{sec}$. The shaded polygon in the figure is the allowable region of the $K c_{1}$ and $K c_{2}$ values.

\footnotetext{
${ }^{1}$ Note that it is proved that under the condition of no internal forces, which is true in our case, $\tilde{G}$ is invertible [15].
} 


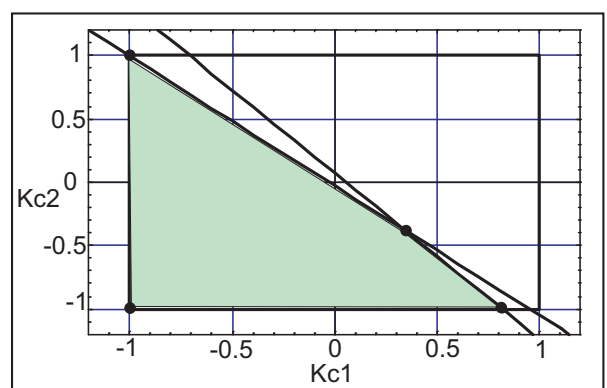

Fig. 5. Combining min-max Kc limits with limits from friction cone constraint,for $\theta=0.2 \mathrm{rad}, \dot{\theta}=1 \mathrm{rad} / \mathrm{sec}$. Vertices give the extreme values.

Srinivasa [15] has proved that such a region must be convex and hence the extremities, $\mathbf{K} \mathbf{c}_{\text {extreme }}$, of this region are at the vertex points of the shaded polygon as shown by the black dots in Figure 5. We can determine the allowable region polygon and the extremities of the allowable region: $\mathbf{K} \mathbf{c}_{\text {extreme }}$, for the range of interest of $\{\mathbf{q}, \dot{\mathbf{q}}\}$. Once we know $\mathbf{K} \mathbf{c}_{\text {extreme }}$ then we can determine $\mathbf{f} \mathbf{c}_{\text {extreme }}$ using Equation 13 and then can determine $\ddot{\mathbf{q}}_{\text {extreme }}$ using Equation 10 for the range of interest of $\{\mathbf{q}, \dot{\mathbf{q}}\}$. Figure 6 shows the plot of the upper and lower limits on $\ddot{\theta}$ for range of values of $\theta$ and $\theta$. Since these regions are determined by incorporating kinematic constraints, contact force constraints and the actuator limits any value of $\{\theta, \dot{\theta}, \ddot{\theta}\}$ inside the region encompassed by the two surfaces is a feasible motion for the system.

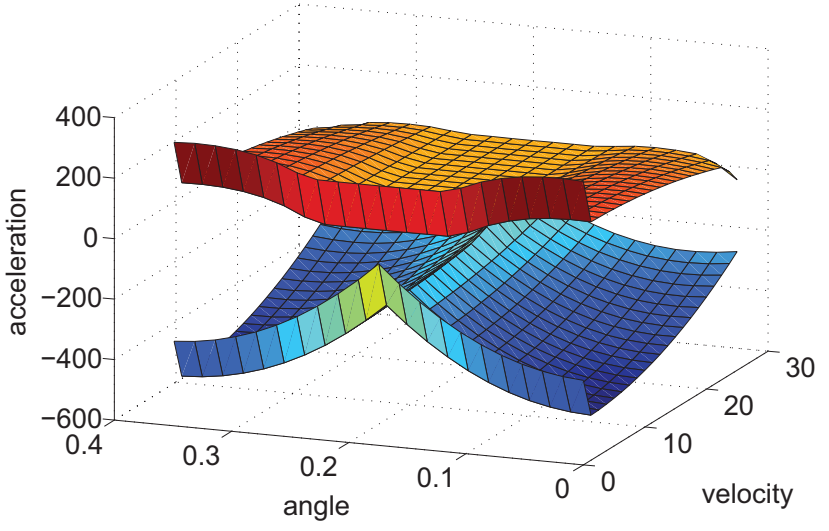

Fig. 6. Limits on acceleration $(\ddot{\theta})$ based on the actuator limits and no-slip condition at various values of rotation angle $(\theta)$ and velocity $(\dot{\theta})$.

\section{Limits on $\ddot{\theta}$ and Control Synthesis}

Determination of $\ddot{\theta}$ limiting surfaces as shown in Figure 6 is extremely useful because based on these limiting surfaces the controller design can be carried out in two stages: design of a trajectory from the start to the goal inside the allowable region, and then design of a feedback controller to follow such trajectory. Optimal control design techniques such as Dynamic programming [1] can be employed to design optimal trajectories going from the start to the goal in the allowable region in the $\{\theta, \dot{\theta}, \ddot{\theta}\}$ space.

Also these limiting surfaces can be pre-computed for the given design parameters of the catoms and can be prefed in the catom controllers. This will drastically reduce the algorithmic complexity which would otherwise arise if we tried to incorporate the dynamics of the catoms in the controller design.

1) Feasibility of a Trajectory: Determination of feasibility of a trajectory from a start location to an end location in $\theta-\dot{\theta}-\ddot{\theta}$ space under the combined constraints represented by the limiting surfaces as shown in Figure 6 is an interesting problem. Many researchers have presented methods to determine feasibility of trajectories from start to goal under constraints in 2D and 3D spaces [10], [12], [14]. For the sake of simplicity we use a sufficiency test for our example. We initiate a trajectory from the start location $\left(\theta_{0}, \ddot{\theta}_{0}\right)$ with maximum allowable $\ddot{\theta}_{\max }$ at that location. And we initiate a backward trajectory from the goal with negative minimum allowable $-\ddot{\theta}_{\min }$ at that location. If these two trajectories intersect, while satisfying the combined constraints represented by $\ddot{\theta}_{\text {min }}-\ddot{\theta}_{\max }$ surfaces, then we know that there is at least one trajectory that reaches from start to goal. Figure 7 a sketch of phase plot with start and goal locations. The arrows at each location represent the acceleration limits based on combined constraints. Figure shows a trajectory starting from start with maximum allowable acceleration and another trajectory from reverse of minimum allowable acceleration. These trajectories satisfy min-max acceleration constraints by staying within the bounds represented by the arrows and intersect, which is a sufficient condition for existing of a feasible trajectory.

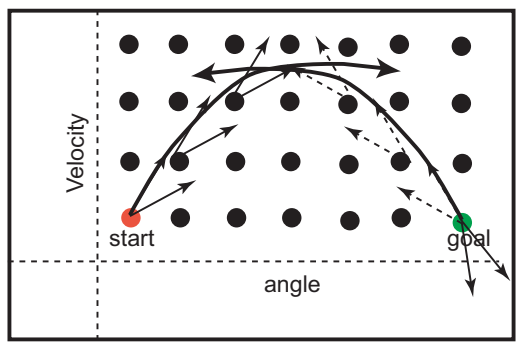

Fig. 7. Determination of feasibility of a trajectory from start to goal under acceleration constraints.

\section{Controller Design and Simulation Results}

We have designed a simple control strategy for the two interacting Catom model using the $\ddot{\theta}$ limit plot. As mentioned earlier, once we have the $\ddot{\theta}$ limit plots the controller design can be divided into two parts: design of a feasible trajectory and then design of a controller to follow this trajectory. We want to design a controller to revolve the rotor from one 


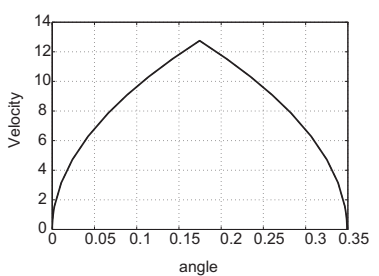

(a) A feasible trajectory

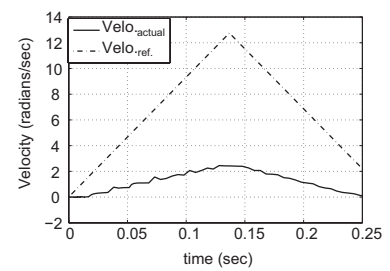

(c) $\dot{\theta}$ tracking

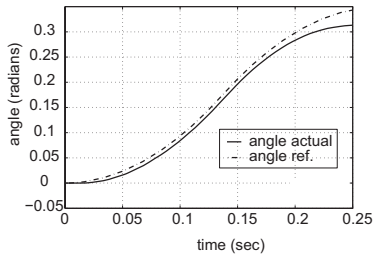

(b) $\theta$ tracking

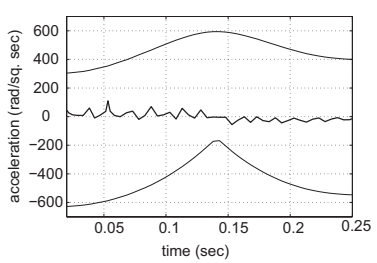

(d) $\ddot{\theta}$ tracking
Fig. 8. Performance of a simple feedback controller which is designed using the allowable volume in the phase space. Controller tried to follow a trajectory given by $8(a)$. Figure shows good tracking performance and also satisfaction of combined constraints

pair of magnets to the next pair, that is, by $\frac{2 \pi}{n_{m}}$ radians. To determine a trajectory, we forward simulate from the start $\{\theta=0, \dot{\theta}=0\}$ with $\ddot{\theta}_{\max }$ at the start and we simulate backward from the goal $\left\{\theta=\frac{2 \pi}{n_{m}}, \dot{\theta}=0\right\}$ with $\ddot{\theta}_{\text {min }}$ at the goal. Our trajectory then is the forward trajectory from the start and then switch over to the trajectory from the goal at the point of intersection. Figure 8(a) shows the trajectory. We then design a simple PID controller based only on the angle feedback to follow along this trajectory.

Figure $8(\mathrm{~b})$ and $8(\mathrm{c})$ the tracking performance of $\theta$ and $\dot{\theta}$, respectively. Figure 8(d) shows variation of $\ddot{\theta}$ and $\ddot{\theta}$ min-max limits as $\theta$ and $\dot{\theta}$ values vary. Note that $\ddot{\theta}$ stays well within the min-max limits thus showing that such controller will work under the given actuator limits as well as the no-slip condition. The goal of this control design exercise is not to present an optimal controller but to demonstrate that once we have the $\ddot{\theta}$ limits from the combined constraints then the controller design can simplified drastically.

\section{E. Stopping Conditions}

As a catom moves around an ensemble it may encounter another catom in its path which will result in an impact. We can check for no slip and no loss of contact conditions under impact by adding the a term representing the impact force in Equation 10. Then we can carry out the analysis as shown above and friction cone condition at contact points between the collided catoms and the ensemble.

\section{Design Guidelines BAsed On COMBInED CONSTRAINTS}

Another important advantage of combining constraints and representing the constraints in the form of min-max surfaces as shown in Figure 6 is that we can use these constraints to derive general guidelines for the catom design. The shape and the size of the $\ddot{\theta}$ plot depends on the physical parameters of the catoms such as the radius, mass, number of magnets, magnet strength etc. and in this section we present some interesting dependencies of $\ddot{\theta}$ limits on the parameter values. Although we do not have analytical relationships for such dependencies we can still determine few useful trends based variations in the $\ddot{\theta}$ limit plots.

Only the volume inside the two surfaces in $\ddot{\theta}$ limit plot is allowable under the no-slip and actuation constraints. Hence, in general, the bigger the volume the better it is for catom motion planning. Figure 9 shows $\ddot{\theta}$ limit plots as we vary the number of magnets and the magnet strength.

- One of the most interesting observations is that as we increase the number of magnet on the catoms the allowable volume increases at first but after a certain value of $n_{m}$ the volume goes down again. This is because there is a trade-off between requirement of normal force and requirement of torque on catoms. This leads to a 'sweet spot' in terms of number of magnets for best catom performance.

- As we reduce the magnet strength the min-max surfaces move closer and at high values of $\dot{\theta}$ start to crop into each other thus reducing the allowable speed. Thus there is a maximum allowable $\dot{\theta}$ associated with given $n_{m}$ and $K_{\max }$.

- At $n_{m}=6$ and at low value of magnet strength ( $K_{\max }<10^{-6} \mathrm{~N}-\mathrm{m}^{3}$ ) the min-max surfaces close in, so much so that there is no feasible value of $\ddot{\theta}$ at $\theta=0$ and $\theta=\frac{2 \pi}{n_{m}}$. Thus it is impossible to move from one magnet pair to the next without loosing contact. This sets a critical lower limit on the number of magnets.

This preliminary analysis shows that our method can be useful to determine the relation between the design parameters to the control limitations. Currently, we are working with hardware development team to use these design guidelines for the design of newer versions of the catoms.

\section{CONCLUSIONS}

In summary, we present a technique for combining the magnet, friction and dynamics constraints in a metamorphic system. The result of the technique is a feasible volume in phase space which encapsulates all of the constraints. We have shown that this volume can be used both to construct motion controllers for the motion of the modules, as well as to compute limits of the design parameters within which feasible motion controllers for the modules must exist.

Furthermore, since this volume, or a parametric representation of the maximal and minimal surfaces, is identical for each module (if the modules are identical), it can be stored 


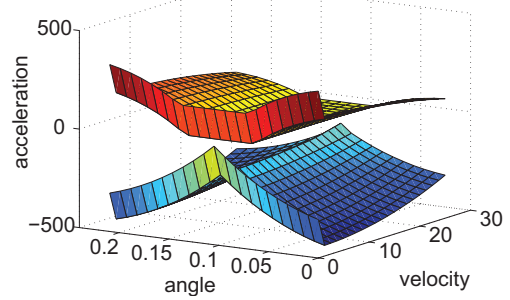

(a) $n_{m}=30, \mathrm{~K}_{\max }=1 * 10^{-5}$

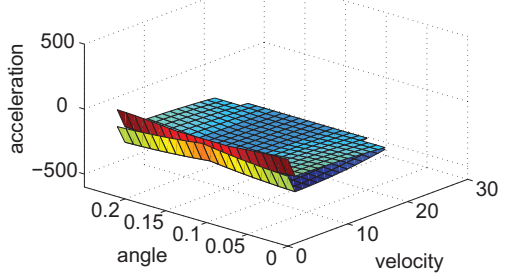

(d) $n_{m}=30, \mathrm{~K}_{\max }=0.2 * 10^{-5}$

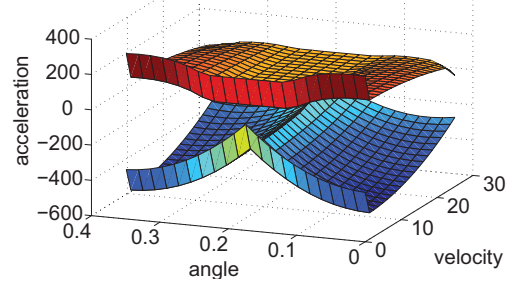

(b) $n_{m}=18, \mathrm{~K}_{\max }=1 * 10^{-5}$

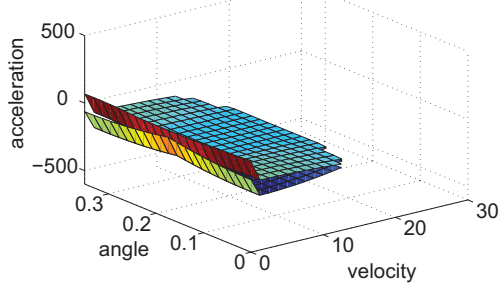

(e) $n_{m}=18, \mathrm{~K}_{\max }=0.210^{-5}$

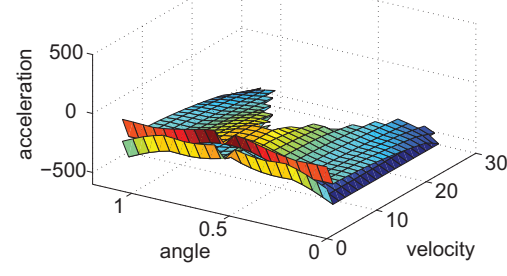

(c) $n_{m}=6, \mathrm{~K}_{\max }=1 * 10^{-5}$

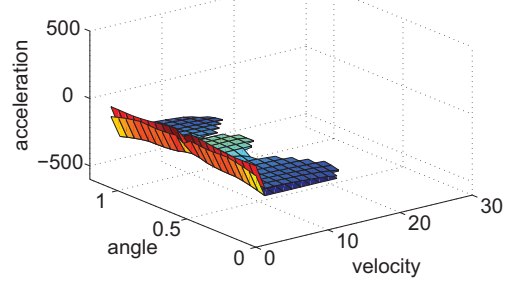

(f) $n_{m}=6, \mathrm{~K}_{\max }=0.2 * 10^{-5}$

Fig. 9. Plots showing acceleration limits $(\ddot{\theta})$ for different as angle $(\theta)$ and velocity $(\dot{\theta})$ are varied. The plots show interesting trends in response to change in $n m$ and $K_{\max }$ values and some general design guidelines can be established based on these trends.

within each module, providing a compact representation of all of the constraints. This representation can be used both for control and for the generation of online dynamically feasible plans.

In the future, we would like to extend our controller design to include robust disturbance rejection, as well as a tolerance to parameter variation. We would also like to relax the assumption of full state observability to a model where the module can only sense a part of its state, for example, its orientation or some coarse measure of it. Furthermore, our controller is designed to provide fairly simple clockwise or counterclockwise rotations to the module, which can be concatenated to construct a dynamic plan. In the future, we would like to incorporate a richer set of possible actions, like the throwing, catching and sliding of modules to construct a wider variety of dynamic plans.

\section{REFERENCES}

[1] Bellman. Dynamic Programming. Princeton University Press, 1957.

[2] P. Bhat, J. Kuffner, S. Goldstein, and S. Srinivasa. Hierarchical motion planning for self-reconfigurable modular robots. In IEEE/RSJ International Conference on Intelligent Robots and Systems, 2006.

[3] A. D. Deshpande and J. Luntz. A unified framework to represent physically cooperating mobile robots and other robotic systems. In Robotics Science and System, 2006.

[4] S. C. Goldstein, J. Campbell, and T. C. Mowry. Exploring programmable matter. IEEE Computer, 2005.

[5] D. T. Greenwood. Principles of Dynamics. Prentice Hall, 1988.

[6] M. W. Jørgensen, E. H. Østergaard, and H. H. Lund. Modular ATRON: Modules for a self-reconfigurable robot. In Proceedings of IEEE/RSJ International Conference on Intelligent Robots and Systems (IROS), pages 2068-2073, 2004.
[7] T. Kane. Dynamics Online: Theory and Implementation with AUTOLEV. OnLine Dynamics, Inc., Sunnyvale, CA, 2000.

[8] B. Khoshnevis, R. Kovac, W.-M. Shen, and P. Will. Reconnectable joints for self-reconfigurable robots. In IEEE/RSJ International Conference on Intelligent Robots and Systems, 2001.

[9] B. Kirby, J. Campbell, B. Aksak, P. Pillai, J. Hoburg, T. Mowry, and S. Goldstein. Catoms: Moving robots without moving parts. In AAAI (Exhibition Abstract), 2005.

[10] M. Mason. Automatic planning of fine motions: Correctness and completeness. In In Proceeding of IEEE Internatinal Conference on Robotics and Automation, 1984.

[11] S. Murata, E. Yoshida, A. Kamimura, H. Kurokawa, K. Tomita, and S. Kokaji. M-TRAN: Self-reconfigurable modular robotic system. IEEE/ASME Transactions on Mechatronics, 7(4):431-441, 2002.

[12] G. Pardo-Castellote and R. H. C. Jr. Proximate time-optimal algorithm for on-line path parameterization and modification. In IEEE International Conference on Robotics and Automation, 1996.

[13] M. D. Rosa, S. Goldstein, P. Lee, J. Campbell, and P. Pillai. Scalable shape sculpting via hole motion. In Proceedings of the 2005 IEEE/RSJ Intl. Conference on Robotics and Automation, 2005.

[14] K. G. Shin and N. D. McKay. A dynamic programming approach to trajectory planning of robotic manipulators. IEEE Transactions on Automatic Control, AC-31:491-500, 1986.

[15] S. Srinivasa. Control synthesis for dynamic contact manipulation. $\mathrm{PhD}$ thesis, Carnegie Melon University, 2005.

[16] S. S. Srinivasa, M. A. Erdmann, and M. T. Mason. Control synthesis for dynamic contact manipulation. In Proceedings of the 2005 IEEE/RSJ Intl. Conference on Robotics and Automation, 2005.

[17] S. S. Srinivasa, M. A. Erdmann, and M. T. Mason. Using projected dynamics to plan dynamic contact manipulation. In Proceedings of the 2005 IEEE/RSJ Intl. Conference on Intelligent Robots and Systems, 2005.

[18] M. Yim, Y. Zhang, K. Roufas, D. Duff, and C. Eldershaw. Connecting and disconnecting for chain self-reconfiguration with polybot. IEEE/ASME Transactions on Mechatronics, special issue on Information Technology in Mechatronics, 7:442-451, 2003. 